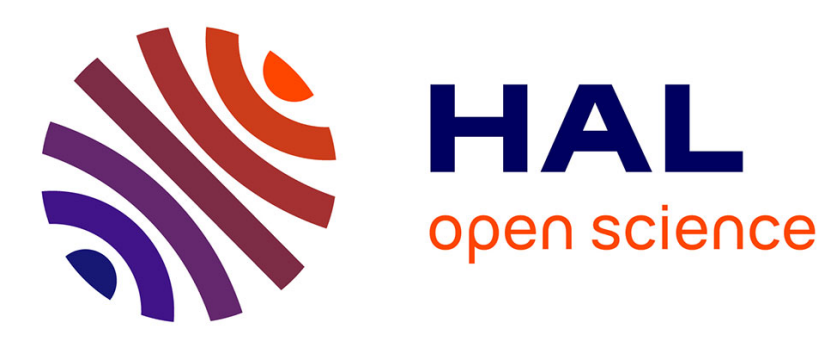

\title{
Parallel Algorithm and Hybrid Regularization for Dynamic PET Reconstruction
}

Nelly Pustelnik, Caroline Chaux, Jean-Christophe Pesquet, Claude Comtat

\section{To cite this version:}

Nelly Pustelnik, Caroline Chaux, Jean-Christophe Pesquet, Claude Comtat. Parallel Algorithm and Hybrid Regularization for Dynamic PET Reconstruction. IEEE Medical Imaging Conference, Oct 2010, Knoxville, Tennessee, United States. pp.2423-2427. hal-00733493

\section{HAL Id: hal-00733493 \\ https://hal.science/hal-00733493}

Submitted on 18 Sep 2012

HAL is a multi-disciplinary open access archive for the deposit and dissemination of scientific research documents, whether they are published or not. The documents may come from teaching and research institutions in France or abroad, or from public or private research centers.
L'archive ouverte pluridisciplinaire HAL, est destinée au dépôt et à la diffusion de documents scientifiques de niveau recherche, publiés ou non, émanant des établissements d'enseignement et de recherche français ou étrangers, des laboratoires publics ou privés. 


\title{
Parallel algorithm and hybrid regularization for dynamic PET reconstruction
}

\author{
N. Pustelnik, Student Member, IEEE, C. Chaux, Member, IEEE, J.-C. Pesquet, Senior Member, IEEE, \\ and C. Comtat, Member, IEEE
}

\begin{abstract}
To improve the estimation at the voxel level in dynamic Positron Emission Tomography (PET) imaging, we propose to develop a convex optimization approach based on a recently proposed parallel proximal method (PPXA). This class of algorithms was successfully employed for 2D deconvolution in the presence of Poisson noise and it is extended here to (dynamic) space + time PET image reconstruction. Hybrid regularization defined as a sum of a total variation and a sparsity measure is considered in this paper. The total variation is applied to each temporal-frame and a wavelet regularization is considered for the space+time data. Total variation allows us to smooth the wavelet artifacts introduced when the wavelet regularization is used alone. The proposed algorithm was evaluated on simulated dynamic fluorodeoxyglucose (FDG) brain data and compared with a regularized Expectation Maximization (EM) reconstruction. From the reconstructed dynamic images, parametric maps of the cerebral metabolic rate of glucose (CMRglu) were computed. Our approach shows a better reconstruction at the voxel level.
\end{abstract}

\section{INTRODUCTION}

The extraction of physiological parameters at the voxel level (parametric imaging) is a challenging problem in dynamic PET imaging (space+time). In [1], [2], [3] the authors demonstrated the advantage of taking into account time-frame characteristics during the reconstruction for improving the image quality and the physiological parameter estimation. More recently, the space+time information was used by considering waveletframe decomposition [3], [4], [5]. To efficiently deal with such representations, the criterion to minimize often involves a $\ell_{1}$ norm (regularization term) which leads to a non-differentiable problem. New convex optimization algorithms (within the class of proximal methods) have been developed in [6], [7] aiming at minimizing criteria which are non-necessarily differentiable. The first application combining wavelet-frame representations and proximal algorithms in dynamic PET image reconstruction was suggested by Verhaeghe et al. [3] by considering Forward-Backward (FB) iterations [6] to minimize a criterion involving a Gaussian data fidelity term and a wavelet spline regularization. In [5], the authors performed Temporal Activity Curves (TAC) denoising in the sinogram space before reconstruction by using the Douglas-Rachford (DR) algorithm so as to deal with a Kullback-Leibler divergence as a data fidelity term, which is well-adapted to Poisson

Manuscript received November 12, 2010.

N. Pustelnik, C. Chaux and J.-C. Pesquet are with the Laboratoire d'Informatique Gaspard Monge, Université Paris-Est, 77454 Marne la Vallée Cedex 2, France (e-mail: \{pustelni,chaux,pesquet\}@univ-mlv.fr).

C. Comtat is with CEA, Service Hospitalier Frédéric Joliot, 91401 Orsay Cedex, France (e-mail: claude.comtat@ cea.fr). noise. To perform simultaneously denoising and reconstruction with wavelet regularization, we previously designed in [8] a nested iterative algorithm (FB-DR algorithm). This method allowed us to improve the reconstruction quality, but its two main drawbacks were (i) a high computational time due to the required subiteration and (ii) the presence of artifacts introduced by wavelet regularization. To tackle these problems, we consider in this paper a more recent algorithm called Parallel ProXimal Algorithm (PPXA) [9] which is well-adapted to dynamic PET due to (i) its parallel structure and (ii) the possibility of dealing with hybrid regularization such as the combination of a wavelet-frame penalty and a total variation measure [10]. This method was presented for image deblurring applications in the presence of Poisson noise [11] and its application to dynamic PET is now investigated.

This paper is organised as follows. First, we will present the considered degradation model and the criterion we aim at minimizing. Then, convex optimization tools such as the proximity operator and PPXA will be introduced. The main technical difficulty in this work consists of computing the proximity operator of the data fidelity term. A solution to overcome this difficulty will be proposed. Finally, numerical experiments will be provided by considering simulated and real dynamic PET $\left[{ }^{18} \mathrm{~F}\right]-\mathrm{FDG}$ exams.

\section{PROBLEM}

We consider a finite parameterization of the original image denoted by $\bar{y}_{t}=\left(\bar{y}_{i, t}\right)_{1 \leq i \leq N}$ which corresponds to the spatial activity distribution for a time-frame $t$. These data are observed through the linear system associated with the matrix $A=\left(A_{j, i}\right)_{1 \leq j \leq M, 1 \leq i \leq N}$ where each element $A_{j, i}$ represents the probability for a voxel $i$ to be detected in the tube of response (TOR) $j$. Moreover, during the acquisition process, data are contaminated by Poisson noise. The effect of the noise is denoted by $\mathcal{P}$. The dynamic PET data $z_{t}=\left(z_{j, t}\right)_{1 \leq j \leq M}$ are related to the original images by the following degradation model:

$$
(\forall t \in\{1, \ldots, T\}) \quad z_{t}=\mathcal{P}\left(A \bar{y}_{t}\right)
$$

where $T$ denotes the number of time-frame. The reconstruction process requires to find the finite parameterization $\hat{y}=\left(\hat{y}_{t}\right)_{1 \leq t \leq T}$ closest to $\bar{y}=\left(\bar{y}_{t}\right)_{1 \leq t \leq T}$ from the measured data $z=\left(z_{t}\right)_{1 \leq t \leq T}$.

In order to perform this task, we consider a variational formulation where the criterion is made up of a data fidelity term related to Poisson noise, a hybrid regularization term 
(total variation + sparsity term in the wavelet-frame domain) and a dynamic range constraint.

The most suited data fidelity term in the presence of Poisson noise is the generalized Kullback-Leibler divergence (related to the Poisson minus log-likelihood) denoted in the following by $D_{\mathrm{KL}}\left(z_{t}, \cdot\right)$, for $z_{t} \in \mathbb{R}^{M}$. Concerning the regularization term, the total variation (denoted by tv) is applied to each time-frame and the wavelet-frame regularization deals simultaneously with the whole space+time data. The total variation allows us to smooth the artifacts introduced by wavelet-frame regularization ( $\ell_{1}$-norm). In the following, $F^{*} \in \mathbb{R}^{N T \times L}$ denotes a general tight wavelet-frame synthesis operator such that $F^{*} \circ F=\nu$ Id for some $\left.\nu \in\right] 0,+\infty[$. Thus, the image $\bar{y}$ can be expressed as $\bar{y}=F^{*} \bar{x}=\left(F_{t}^{*} \bar{x}\right)_{1 \leq t \leq T}$, where $\bar{x} \in \mathbb{R}^{L}$ denotes the wavelet-frame coefficients and $F_{t}^{*} \in \mathbb{R}^{N \times L}$. The convex constraint $C \subset \mathbb{R}^{N T}$ limits the data value range of the solution (for example, it may be a positivity constraint). Below, $\iota_{C}$ denotes the indicator function of $C$ such that, for every $y \in \mathbb{R}^{N T}, \iota_{C}(y)=0$ if $y \in C$ and $+\infty$ otherwise. The resulting minimization problem amounts to finding the wavelet-frame coefficients such that

$$
\begin{array}{r}
\hat{x} \in \underset{x \in \mathbb{R}^{L}}{\operatorname{Argmin}} \sum_{t=1}^{T}\left(D_{\mathrm{KL}}\left(z_{t}, A F_{t}^{*} x\right)+\vartheta \operatorname{tv}\left(F_{t}^{*} x\right)\right) \\
+\kappa\|x\|_{1}+\iota_{C}\left(F^{*} x\right) .
\end{array}
$$

$\vartheta>0$ and $\gamma>0$ denote the regularization parameters. They allow us to adjust the impact of the total variation and sparsity penalties on the solution. The reconstructed image is $\hat{y}=F^{*} \hat{x}$. Due to the definition of each functional, this criterion is convex but non-differentable. To solve efficiently such a minimization problem, we will consider recent convex optimization tools.

\section{CONVEX OPTIMIZATION}

One of the most famous convex optimization algorithm is the Projection Onto Convex Sets (POCS) algorithm [12] based on alternated projections. A generalization of projections was proposed by Moreau in [13], which is known as the proximity operator. This tool is defined for a convex, lower semi-continuous and proper function $\left.\left.\varphi: \mathbb{R}^{X} \rightarrow\right]-\infty,+\infty\right]$, at a point $v \in \mathbb{R}^{X}$ and it is such that

$$
\operatorname{prox}_{\varphi}: \mathbb{R}^{X} \rightarrow \mathbb{R}^{X}: v \mapsto \arg \min _{u \in \mathbb{R}^{X}} \frac{1}{2}\|u-v\|^{2}+\varphi(u) .
$$

Particularly, if $C$ is a nonempty closed convex set of $\mathbb{R}^{X}$, $\operatorname{prox}_{\iota_{C}}$ reduces to the projection $P_{C}$ onto $C$.

In the recent literature, proximal algorithms (that is based on proximity operators) have been proposed to minimize efficiently non-differentiable convex criteria. One of the most famous ones is the FB algorithm [14] also known as thresholded Landweber or iterative soft-thresholding [6] when a $\ell_{1}+\ell_{2}$ criterion is considered. More generally, the FB algorithm allows us to minimize a sum of two functions one of which is Lipschitz differentiable. This framework is however too restrictive to solve the minimization problem (2) due to the non-Lipschitz differentiability of $D_{\mathrm{KL}}$ [7]. Another proximal algorithm known as PPXA [9] allows us to relax the Lipschitz assumption and to solve any minimization problem formulated as

$$
\hat{x} \in \underset{x \in \mathbb{R}^{L}}{\operatorname{argmin}} \sum_{q=1}^{Q} f_{q}(x)
$$

where $Q \in \mathbb{N}^{*}$ and where $\left(f_{q}\right)_{1 \leq q \leq Q}$ are convex, lower semicontinuous and proper functions from $\mathbb{R}^{L}$ to $\left.]-\infty,+\infty\right]$. The iterations are recalled in Algorithm 1.

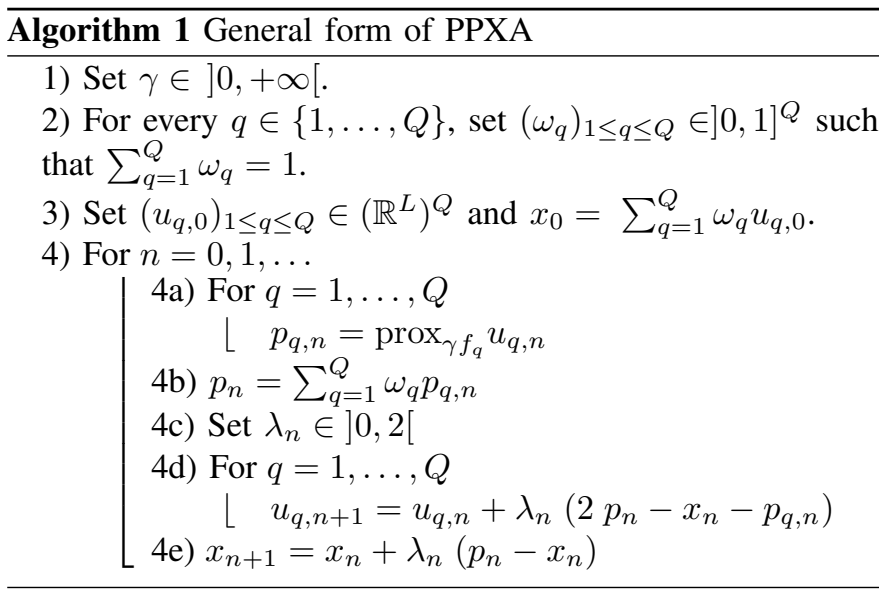

The convergence of the sequence $\left(x_{n}\right)_{n \in \mathbb{N}}$ generated by Algorithm 1 to a solution $\widehat{x}$ of (3) is established under the following assumption.

\section{Assumption III.1}

(i) $\lim _{\|x\| \rightarrow+\infty} f_{1}(x)+\cdots+f_{Q}(x)=+\infty$.

(ii) $\bigcap_{q=1}^{Q} \operatorname{rint} \operatorname{dom} f_{q} \neq \varnothing$. $^{1}$

(iii) $\sum_{n \in \mathbb{N}} \lambda_{n}\left(2-\lambda_{n}\right)=+\infty$.

The main difficulty of this algorithm lies in Step 4a) which requires to compute the proximity operator of each function $f_{q}$. Fortunately, for a large class of functions, this operator takes a closed form [14]. For example, the $\ell_{1}$-norm proximity operator corresponds to a soft-thresholding. The way to proceed with tv is detailed in [9]: it results that tv can be split in a sum of four functions, denoted by $\left(\operatorname{tv}_{i}\right)_{1 \leq i \leq 4}$ when Haar filters are considered. However, a remaining difficulty in using this algorithm for dynamic PET reconstruction is to obtain an explicit form at Step 4a) for $D_{\mathrm{KL}}\left(z_{t}, A \cdot\right)$.

Subsequently, we assume that for every $r \in\{1, \ldots, R\}$, $\mathbb{I}_{r}$ is a partition of $\{1, \ldots, M\}$ in nonempty sets. Additionally, we can write $D_{\mathrm{KL}}\left(z_{t}, \cdot\right)$ as a sum of $M$ functions $\left.\left.\Psi_{j, t}: \mathbb{R} \rightarrow\right]-\infty,+\infty\right]$ operating on each $j$-th component $z_{j, t}$ of $z_{t}$. By regrouping the terms in this sum such that $j \in \mathbb{I}_{r}$ (for every $\left.r \in\{1, \ldots, R\}, \Psi_{t}^{(r)}\left(\left(z_{j, t}\right)_{j \in \mathbb{I}_{r}}\right)=\sum_{j \in \mathbb{I}_{r}} \Psi_{j, t}\left(z_{j, t}\right)\right)$, $D_{\mathrm{KL}}\left(z_{t}, A\right.$.) can be decomposed as a sum of $R$ functions involving reduced-size linear operators $A^{(r)}=\left(A_{j, i}\right)_{j \in \mathbb{I}_{r}, 1 \leq i \leq N}$ containing non-overlapping and thus orthogonal rows of $A$. For every $r \in\{1, \ldots, R\}, \mathbb{I}_{r}$ denotes a set of TOR involving distinct pixels. The resulting PPXA iterations are detailed in Algorithm 2.

\footnotetext{
${ }^{1}$ Let $\left.\left.\varphi: \mathbb{R}^{X} \rightarrow\right]-\infty,+\infty\right]$ be a convex, 1.s.c. and proper function. $\operatorname{dom} \varphi=\left\{u \in \mathbb{R}^{X} \mid \varphi(u)<+\infty\right\}$ and the interior of $C \subset \mathbb{R}^{X}$ relative to its affine hull is the set denoted by $\operatorname{rint} C$.
} 


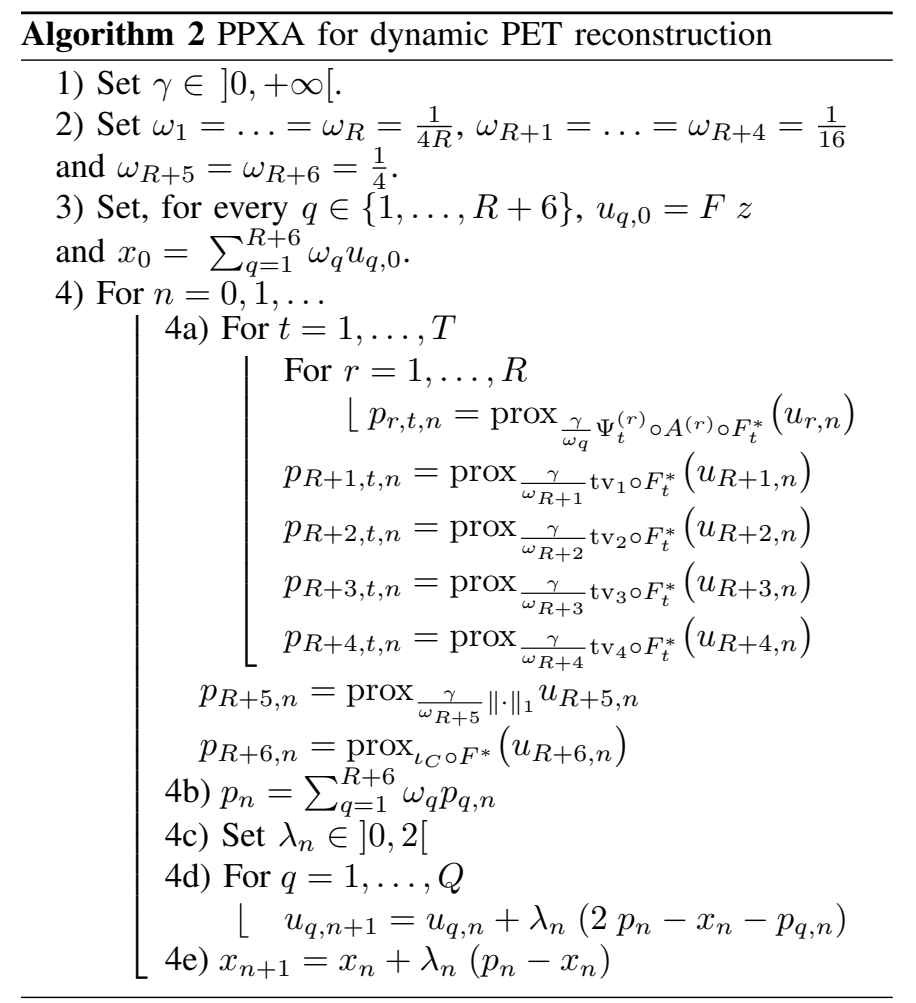

\section{Materials And Methods}

\section{A. Simulated data}

The method was evaluated on simulated 2D+time data, using one slice of the Zubal brain phantom [15]. A two compartment model with $k_{4}=0$ was used to simulate a dynamic [18F]-FDG brain study, with 16 time frames with a duration varying between 30 seconds for the first time-frames to 5 minutes for the last ones. Moreover, small local variations of $K_{i}$ were created in the cortex and putamen (see Figure 2, top-left). 288 (radial) $\times 144$ (angles) sinograms with a radial sampling of $2.247 \mathrm{~mm}$ were simulated by analytically projecting the phantom and adding Poisson noise. Attenuation, random and scattered coincidences were not simulated in this example. The number of events varies from 48 for the first time-frame to 26804 for the last time-frame.

The PPXA algorithm is implemented in parallel with the OpenMP library and run over 400 iterations of 10 s each. The relaxation parameter is here equal to $\lambda_{n} \equiv 1$ and the stepsize is $\gamma=6.10^{5}$. The system matrix is split in $R=432$ subsets and the function number is $Q=R+6$. The chosen separable orthonormal wavelets correspond to symmlet filters of length 6 over 3 resolution levels of spatial decomposition. PPXA 1 refers to our approach without temporal regularization and PPXA 2 considers a two-level decomposition with Daubechies-6 wavelets on the interval for temporal decomposition [16]. The latter choice is motived by the small number of time-frames and aims at avoiding boundary effects. The constraint on the data range is $C=\left[0,10^{5}\right]^{N T} \mathrm{~Bq} / \mathrm{cc}$ and the chosen regularization parameters are $\kappa=0.1$ and $\vartheta=10^{-5}$. A limited value of the parameter $\vartheta$ was chosen so as to avoid "cartoon effects". The proposed solution without/with temporal regularization in PPXA 1/PPXA 2 is compared with EM-ML and EM-ML with post-smoothing (Sieves). SIEVES 1 denotes a Gaussian filtering optimized to yield the minimum MSE and SIEVES 2 uses a Gaussian kernel leading to a similar noise level than our approach. For EM-ML and SIEVES 1/2, the iteration number is 250 . A full width at half maximum (FWHM) of $12 \mathrm{~mm} \times 12 \mathrm{~mm}$ (resp. $14 \mathrm{~mm} \times 14 \mathrm{~mm}$ ) is used for SIEVES 1 (resp. SIEVES 2).

\section{B. Pre-clinical data}

We also processed real data resulting from a $\left[{ }^{18} \mathrm{~F}\right]-\mathrm{FDG}$ exam on the ecat HR+ scanner (Siemens medical solutions, Knoxville, TN, USA). $284 \mathrm{MBq}$ were injected to a $4.5 \mathrm{~kg}$ baboon. The injection was done under camera. A dynamic acquisition was performed with septa extended. The prompt and delayed coincidences were registered separately in 16 frames with a duration varying from 1.5 minutes to 4 minutes. The global exam duration was 54 minutes. Transmission was realized before injection by a rotative source of germanium 57 .

We reconstructed prompt data by considering three different approaches: filtered backprojection (FBP), EM-ML reconstruction with Gaussian (12 $\mathrm{mm} \times 12 \mathrm{~mm}$ FWHM) postfiltering (Sieves) and our approach (PPXA). During the reconstruction process, attenuation effects, normalization effects, and random coincidences have been taken into account. However, scattered coincidences have not been considered (this choice is justified by the septa extended). The system matrix used for SIEVES and PPXA does not model resolution (PSF) effects.

\section{REsults}

\section{A. Simulated data}

Figures 1 and 2 display the reconstruction results for different temporal-frames ( $t=4: 1.5-2 \mathrm{~min}$ and $t=7: 8-13 \mathrm{~min}$ ). During first frames when the noise level is particularly high, PPXA 2 restores quite well the brain activity even if artifacts can be observed (for example in the thalamus area).

Table I compares the normalized MSE for different areas of interest such as the cortex, the thalamus and the striatum. It can be observed that PPXA 2 leads to the smallest normalized MSE for the first frames and equivalent normalized MSE for the last ones.

Figures 3, 4, and 5 represent Temporal Activity Curves for individual pixels in different area of interest. This allows us to evaluate the impact of the spatio-temporal reconstruction and the good results obtained with PPXA 2.

Using Pmod software (PMOD Technologies Ltd, Zürich, CH), parametric images of CMRglu were computed from the EMML, Sieves, and PPXA reconstructions. The results are shown in Figure 6. The differences between the CMRglu images is not as much important as the TAC results or the reconstructed images. This is mainly due to the Patlak model which essentially uses the last frames for CMRglu computation, which are less sensitive to noise. 

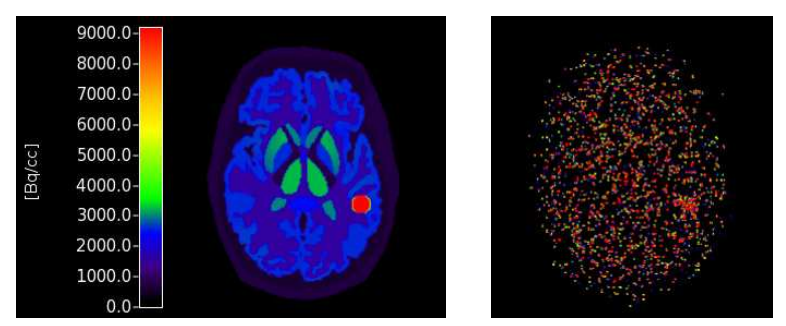

Original

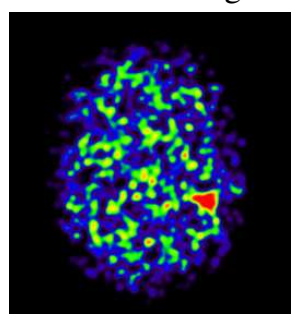

SIEVES 1

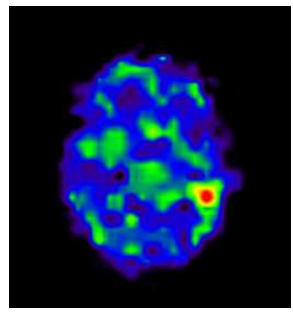

PPXA 1

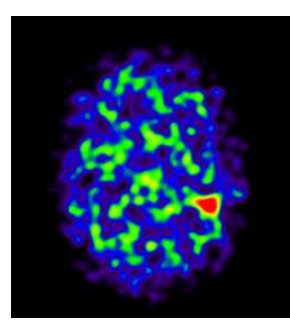

SIEVES 2

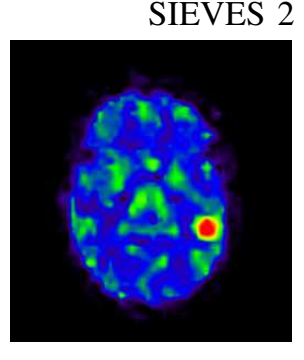

PPXA 2

Fig. 1. Reconstructed images (in $\mathrm{Bq} / \mathrm{cc}$ ) for $t=4$

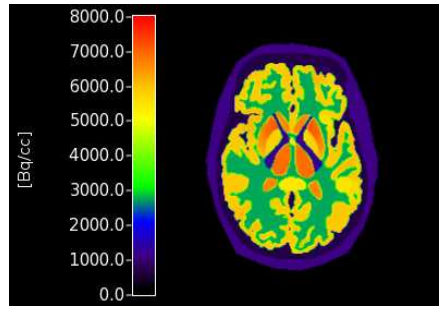

Original

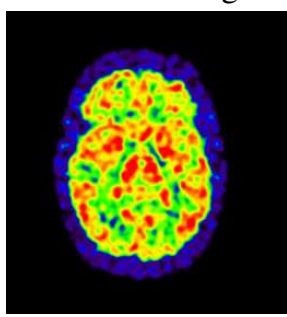

SIEVES 1

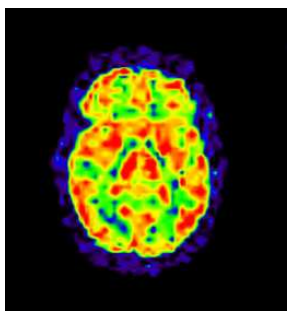

PPXA 1

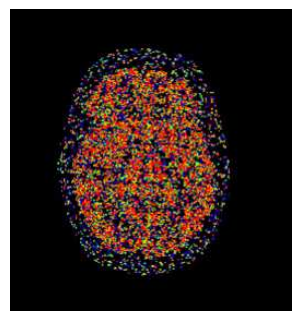

EM-ML

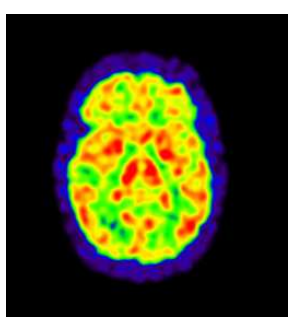

SIEVES 2

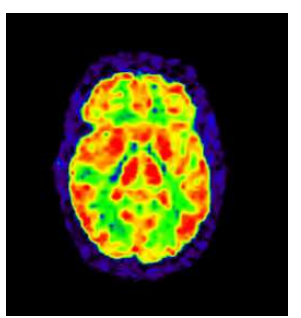

PPXA 2

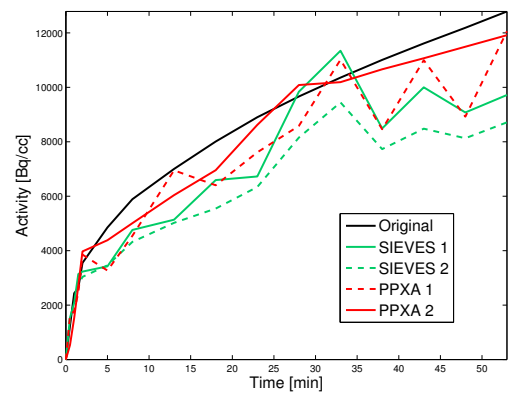

Pixel number : $(86,170)$

Fig. 4. TAC in the cortex.

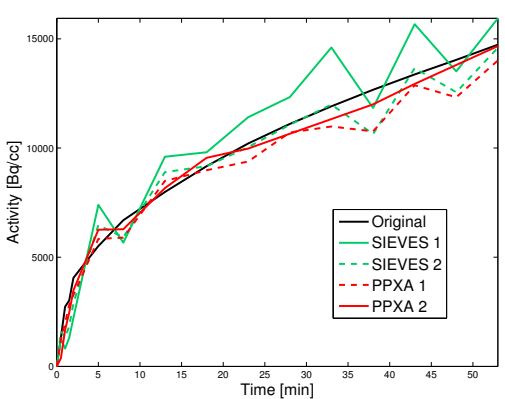

Pixel number : $(101,120)$

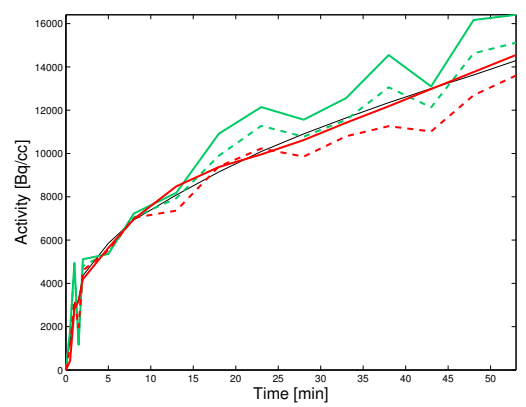

Pixel number : $(124,120)$

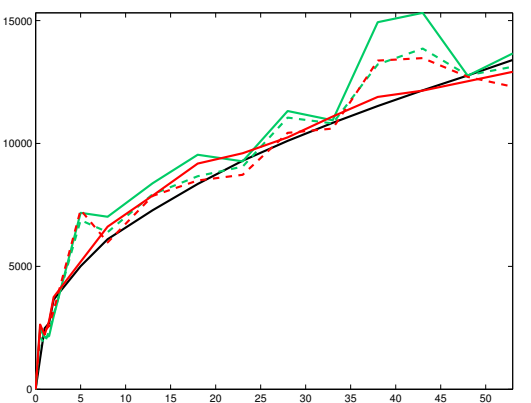

Pixel number : $(106,92)$

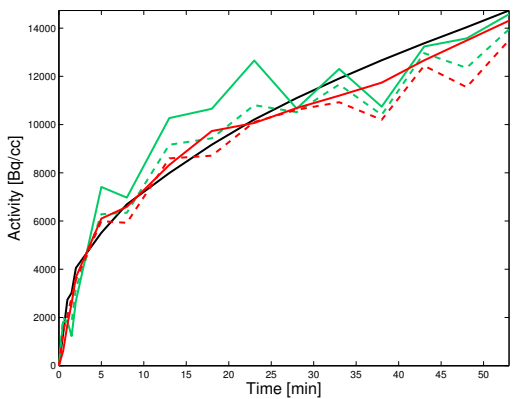

Pixel number : $(103,121)$

Fig. 2. Reconstructed images (in $\mathrm{Bq} / \mathrm{cc}$ ) for $t=7$.

Fig. 5. TAC in the striatum. 


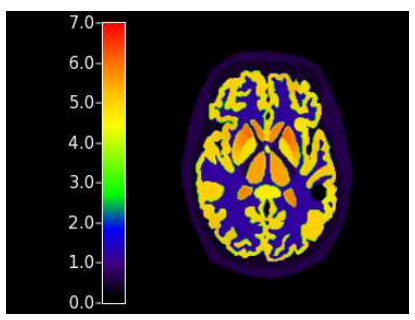

Original

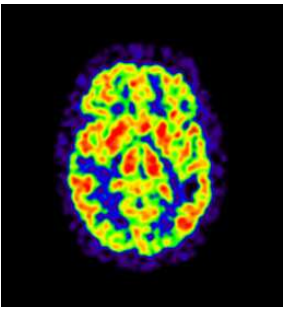

SIEVES 1

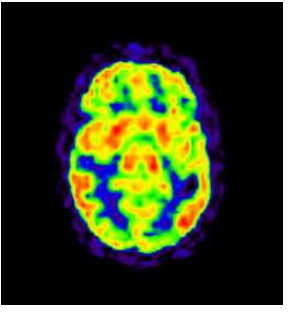

PPXA 1

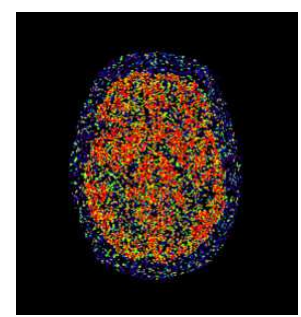

EM-ML

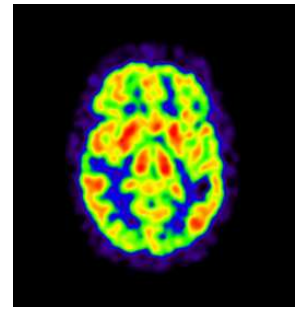

SIEVES 2

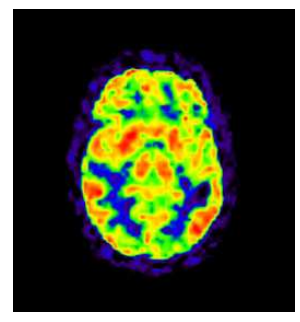

PPXA 2
Fig. 6. CMRglu computed from the Original, EM-ML, Sieves, and PPXA reconstructed images (in $\mathrm{mol} / \mathrm{min} / 100 \mathrm{~g}$ ).

\section{B. Pre-clinical data}

Figure 7 shows the reconstructed images obtained with the different reconstruction methods for temporal-frames $t=4$ (4-6 $\mathrm{min}), t=7$ (14-18 $\mathrm{min})$, and $t=14$ (42-46 $\mathrm{min})$. These results illustrate the good behaviour and the quantitative correctness of the proposed method on real data.

\section{CONCLUSION}

We have presented a convex optimization approach to deal with spatio-temporal PET reconstruction. The minimized criterion is composed with different terms: generalized KullbackLeibler divergence (minus Poisson log-likelihood) term, $\ell_{1}$ norm for wavelet-based regularization, total variation and dynamic range constraint. To minimize efficiently this criterion, we employed PPXA which is a recent algorithm belonging to the class of proximal methods. The convergence of this algorithm is guaranteed and it is implementable in parallel. The effectiveness of the proposed approach was demonstrated on simulated and real pre-clinical data.

\section{REFERENCES}

[1] T. E. Nichols, J. Qi, E. Asma, and R. M. Leahy, "Spatiotemporal reconstruction of list-mode PET data," IEEE Trans. Med. Imag., vol. 21, no. 4, pp. 396-404, 2002.

[2] M. E. Kamasac, C. A. Bouman, E. D. Morris, and K. Sauer, "Direct reconstruction of kinetic parameter images from dynamic PET data," IEEE Trans. Med. Imag., vol. 24, no. 6, pp. 636-650, 2005.
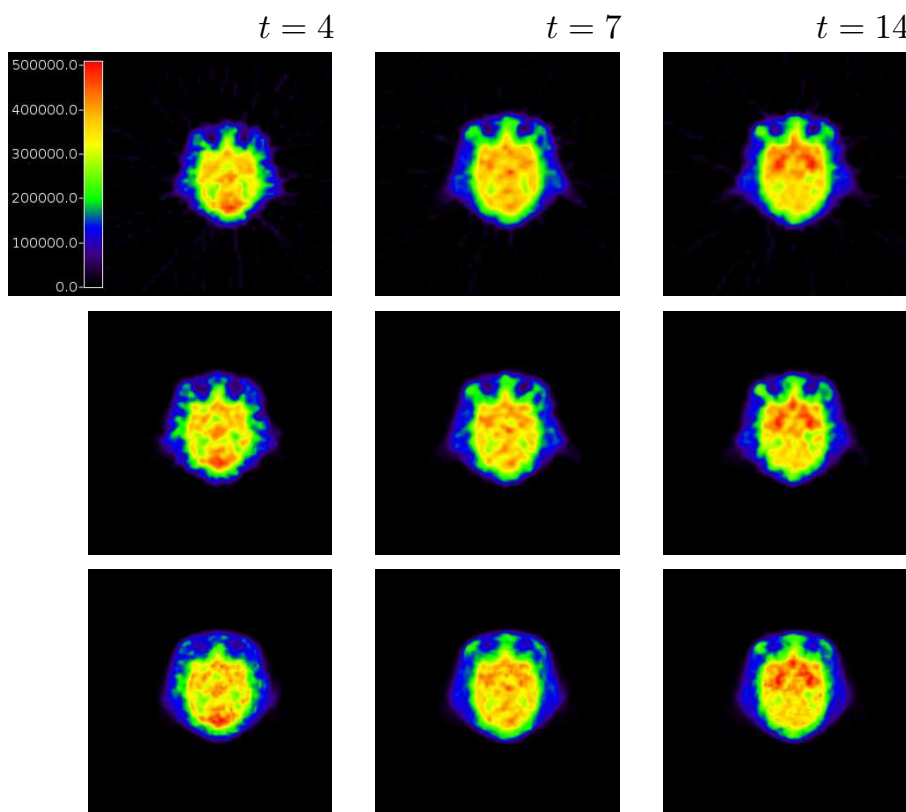

Fig. 7. Reconstructed activity (in Bq/cc). 1st row : SIEMENS, 2nd row : EM-ML with post-smoothing (Sieves), 3rd row : PPXA.

[3] J. Verhaeghe, D. Van De Ville, I. Khalidov, Y. D'Asseler, I. Lemahieu, and M. Unser, "Dynamic PET reconstruction using wavelet regularization with adapted basis functions," IEEE Trans. Med. Imag., vol. 27, no. 7, pp. 943-959, July 2008.

[4] J. Zhou, L. Senhadji, J.-L. Coatrieux, and L. Luo, "Iterative PET image reconstruction using translation invariant wavelet transform," IEEE Trans Nucl. Sci., vol. 56, no. 1, pp. 116-128, Feb. 2009.

[5] F. C. Sureau, J.-C. Pesquet, C. Chaux, N. Pustelnik, A. J. Reader, C. Comtat, and R. Trebossen, "Temporal wavelet denoising of PET sinograms and images," in Nuclear Science Symposium and Medical Imaging Conference Record, Dresden, Germany, 19-25 Oct. 2008, pp. 4448-4454.

[6] I. Daubechies, M. Defrise, and C. De Mol, "An iterative thresholding algorithm for linear inverse problems with a sparsity constraint," Comm. Pure Applied Math., vol. 57, no. 11, pp. 1413-1457, Nov. 2004.

[7] C. Chaux, J.-C. Pesquet, and N. Pustelnik, "Nested iterative algorithms for convex constrained image recovery problems," SIAM J. Imag. Sc., vol. 2, no. 2, pp. 730-762, June 2009.

[8] N. Pustelnik, C. Chaux, J.-C. Pesquet, F. C. Sureau, E. Dusch, and C. Comtat, "Adapted convex optimization algorithm for wavelet-based dynamic PET reconstruction," in the proceeding of the 10th International Meeting on Fully Three-Dimensional Image Reconstruction in Radiology and Nuclear Medicin, Beijing, China, 5-10 Sept. 2009.

[9] P. L. Combettes and J.-C. Pesquet, "A proximal decomposition method for solving convex variational inverse problems," Inverse Probl., vol. 24, no. 6 , pp. $x+27$, Dec. 2008.

[10] L. Rudin, S. Osher, and E. Fatemi, "Nonlinear total variation based noise removal algorithms," Physica D, vol. 60, no. 1-4, pp. 259-268, Nov. 1992.

[11] N. Pustelnik, C. Chaux, and J.-C. Pesquet, "Parallel ProXimal Algorithm for image restoration using hybrid regularization," 2010, submitted.

[12] L. M. Bregman, "The method of successive projection for a common point of convex sets," Soviet Math. Dokl., vol. 6, pp. 688-692, 1965.

[13] J. J. Moreau, "Proximité et dualité dans un espace hilbertien," Bull. Soc. Math. France, vol. 93, pp. 273-299, 1965.

[14] C. Chaux, P. L. Combettes, J.-C. Pesquet, and V. R. Wajs, "A variational formulation for frame-based inverse problems," Inverse Probl., vol. 23 , no. 4, pp. 1495-1518, June 2007.

[15] I. G. Zubal, C. R. Harrell, E. O. Smith, Z. Rattner, G. Gindi, and P. B. Hoffer, "Computerized three-dimensional segmented human anatomy," Medical Physics, vol. 21, no. 1, pp. 299-302, 1994.

[16] A. Cohen, I. Daubechies, and P. Vial, "Wavelets on interval and fast wavelet transforms," Appl. and Comp. Harmonic Analysis, vol. 1, no. 1, pp. 54-81, 1993 